\title{
First detection and genetic characterisation of Enterocytozoon bieneusi in wild deer in Melbourne's water catchments in Australia
}

\author{
Yan Zhang ${ }^{1}$, Anson V. Koehler ${ }^{1 *}$, Tao Wang ${ }^{1}$, Shane R. Haydon² and Robin B. Gasser ${ }^{1 *}$
}

\begin{abstract}
Background: Enterocytozoon bieneusi is reported to be a common microsporidian of humans and animals in various countries. However, E. bieneusi has yet to be recorded in animals in Australia. Here, we undertook the first molecular epidemiological investigation of E. bieneusi in three species of deer (Cervus elaphus, Dama dama and Rusa unicolor) that live in the catchment areas that supply the city of Melbourne with drinking water.

Methods: Genomic DNA was extracted from a total of 610 individual faecal samples from wild deer, including sambar deer (Rusa unicolor) ( $n=516)$, red deer (Cervus elaphus) $(n=77)$ and fallow deer (Dama dama) $(n=17)$ from nine catchment areas, and then tested using a nested PCR-based sequencing approach employing internal transcribed spacer (ITS) of nuclear ribosomal DNA as the genetic marker.

Results: Enterocytozoon bieneusi was detected in 25 of all 610 (4.1\%) samples exclusively in samples from sambar deer. The analysis of ITS sequence data revealed three known ( $D, J$ and Type IV) and two new (MWC_d1 and MWC_d2) genotypes of E. bieneusi. Although the significance of the latter two new genotypes is presently unknown, phylogenetic analysis of ITS sequence data sets showed that they cluster with genotypes D and Type IV, which have been recorded previously in humans. These findings suggest that sambar deer in the water catchments harbour zoonotic genotypes of E. bieneusi.

Conclusions: Further insight into the epidemiology of E. bieneusi in wildlife, water and the environment in Australia will be important to have an informed position on the public health significance of microsporidiosis caused by this microbe.
\end{abstract}

Keywords: Enterocytozoon bieneusi, Genotypes, Internal transcribed spacer (ITS) of nuclear ribosomal DNA, Prevalence, Sambar deer, Australia

\section{Background}

Enterocytozoon bieneusi is a member of the Microsporidia [1-3] and is recognised as a fungus, although its exact classification is currently being discussed [4-8]. More than 1200 microsporidia have been recorded from a range of animals species [9]. Currently, 17 microsporidian species are recognised to infect humans, of which E. bieneusi is most commonly found to be pathogenic [10].

Enterocytozoon bieneusi was first detected in a Haitian patient with HIV/AIDS who suffered from severe diarrhoea

\footnotetext{
* Correspondence: anson.koehler@unimelb.edu.au; robinbg@unimelb.edu.au ${ }^{1}$ Department of Veterinary Biosciences, Melbourne Veterinary School, Faculty of Veterinary and Agricultural Sciences, The University of Melbourne, Parkville, VIC 3010, Australia

Full list of author information is available at the end of the article
}

[11]. Numerous published reports (reviewed in $[10,12,13]$ ) show that this species can infect both immunocompromised and immunocompetent people. Enterocytozoon bieneusi is often an opportunistic pathogen in the former group of humans [12, 14-17], typically causing mild to severe, acute or chronic diarrhoea, malabsorption and/or wasting [18-21]. Some individuals infected with E. bieneusi do not show clinical signs and thus represent carriers of this microsporidian, with the potential of spreading spores to the environment and/or other people or animals, which is an important epidemiological consideration [1, 22-24]. Humans and animals are usually infected via a faecal-oral transmission route, either through spore-contaminated water $[25,26]$ or food $[27,28]$. Interestingly, E. bieneusi has 
also been detected in the respiratory tracts of AIDS patients; however, whether E. bieneusi is capable of airborne transmission requires verification [29-31]. Nonetheless, a study has reported that $E$. bieneusi spores can be found in air and could potentially be inhaled by humans [32].

Enterocytozoon bieneusi isolates are usually characterised genetically by sequencing their internal transcribed spacer region (ITS) of nuclear ribosomal DNA. To date, more than 200 distinct genotypes have been reported in the literature (reviewed in [10,12]). Some of them appear to be host-specific or host-adapted. However, others can be found in different species of animals as well as humans, indicating the zoonotic potential of these E. bieneusi genotypes (e.g. D, EbpA, J, K and Type IV; [12]). Thus, the National Institute of Allergy and Infectious Diseases (NIAID) has classified E. bieneusi as a Category B Priority Pathogen [33].

Various studies [34-51] have assessed the diversity of genotypes of $E$. bieneusi in a broad range of wild and domestic animal hosts, including mammals (carnivores, lagomorphs, primates, rodents and ungulates) and birds, in as many as 39 countries. However, almost nothing is known about the transmission routes of most genotypes or their zoonotic capacity. Moreover, with the exception of records of $E$. bieneusi in HIV/AIDS patients [14, 52], surprisingly, there is no published record of E. bieneusi in any species of animals from Australia. The present study takes a first step to investigating E. bieneusi in animals in Australia. Using a molecular approach, we explored the presence and distribution of this microsporidian in wild deer inhabiting catchment areas (managed by the Melbourne Water Corporation, MWC) that supply people in the city of Melbourne and environs with treated (but unfiltered) drinking water.

\section{Methods}

\section{Melbourne's water catchments}

Ten main water catchment reservoirs (www.melbournewater.com.au) supply drinking water to the people of Melbourne and environs. Approximately $80 \%$ of Melbourne's drinking water is drawn from 'closed' catchments in the Yarra Ranges (east of Melbourne). These catchments cover almost 160,000 ha of eucalypt forest. Access by humans and domestic animals is restricted. The remaining $20 \%$ of Melbourne's water comes from 'open' catchments, in which some farming and human activities are permitted. All water undergoes treatment, in accordance with national and international guidelines [53, 54]. The nine catchment areas studied here are located north and east of Melbourne, are less than $90 \mathrm{~km}$ apart and include: Armstrong (AM) $37^{\circ} 38^{\prime} \mathrm{S} 145^{\circ} 51^{\prime} \mathrm{E}$; Cardinia (CA) $37^{\circ} 47^{\prime} \mathrm{S} 145^{\circ}$ 24'E; Maroondah (MR) 37² $38^{\prime} \mathrm{S} 145^{\circ} 33^{\prime} \mathrm{E}$; O'Shannassy (OS) $37^{\circ} 40^{\prime} \mathrm{S} \quad 145^{\circ} 48^{\prime} \mathrm{E}$; Silvan (SV) $37^{\circ} 50^{\prime} \mathrm{S} \quad 145^{\circ} 25^{\prime} \mathrm{E}$; Tarago (TAR) $37^{\circ} 59^{\prime} \mathrm{S} 145^{\circ} 55^{\prime} \mathrm{E}$; Thompson (TH) $37^{\circ} 47^{\prime} \mathrm{S}$ $146^{\circ} 21^{\prime} \mathrm{E}$; Upper Yarra (UY) $37^{\circ} 40^{\prime} \mathrm{S} 145^{\circ} 55^{\prime} \mathrm{E}$ and Yan Yean (YY) $37^{\circ} 33^{\prime} \mathrm{S} 145^{\circ} 08^{\prime} \mathrm{E}[55,56]$. Reservoirs MR, OS, TH and UY are situated in the densely forested Yarra Ranges catchment, whereas the YY reservoir is a much smaller catchment north of Melbourne and surrounded by residential and grazing land. The other reservoirs, including CA and SV, act as storage facilities for the larger catchments, and have eucalypt and/or pine forests. TAR is the one 'open' water-supply catchment where farming in the land surrounding the reservoir is permitted. Small areas of grassland abut some water reservoirs, from where faecal samples were collected in this study. Most of the faecal samples from deer collected here originate from catchment areas CA, MR, OS, UY and YY.

\section{Samples and DNA isolation}

A total of 610 faecal samples from wild deer, including sambar deer (Rusa unicolor; $n=516$ ), red deer (Cervus elaphus; $n=77$ ) and fallow deer (Dama dama; $n=17$ ), were collected from nine of Melbourne's water catchments from June 2009 to March 2017. Specifically, samples were collected from AM $(n=12)$, CA $(n=114)$, MR $(n=98)$, OS $(n=112), \mathrm{SV}(n=3)$, TAR $(n=16), \mathrm{TH}(n=$ $8)$, UY $(n=134)$ and YY $(n=113)$. Genomic DNA was extracted directly from $0.2 \mathrm{~g}$ of each of the 610 faecal samples using the PowerSoil kit (MoBio, Carlsbad, CA, USA), according to the manufacturer's instructions, and stored at $-20{ }^{\circ} \mathrm{C}$. This kit was used, because it is highly effective at removing components from faecal samples that are inhibitory to PCR [56]. Scats were initially identified using a field guide [57], and host identity was unequivocally confirmed by testing faecal DNA using a nested PCR-based sequencing approach employing the mitochondrial cytochrome $b$ gene (cf. [58]).

\section{Nested PCR-based sequencing of E. bieneusi ITS}

From individual faecal DNA samples, the internal transcribed spacer (ITS) of nuclear ribosomal DNA of E. bieneusi was specifically amplified by PCR using degenerate primers originally designed and evaluated by KatzwinkelWladarsch et al. [59]. In the first PCR round, primers MSP-1 (forward: 5' -TGA ATG KGT CCC TGT-3') and MSP-2B (reverse: 5'-GTT CAT TCG CAC TAC T-3') were used to amplify $601 \mathrm{bp}$ of ITS plus flanking gene sequences. In the second round, primers MSP-3 (forward: 5'-GGA ATT CAC ACC GCC CGT CRY TAT-3') and MSP-4B (reverse: $5^{\prime}$-CCA AGC TTA TGC TTA AGT CCA GGG AG-3') were employed to amplify a product of $535 \mathrm{bp}$ containing $130 \mathrm{bp}$ of the 3 '-end of the small subunit $(S S U)$ of the nuclear rRNA gene, $243 \mathrm{bp}$ of the ITS and $162 \mathrm{bp}$ of the $5^{\prime}$-region of the large subunit $(L S U)$ rRNA gene.

Nested PCR (in $50 \mu \mathrm{l}$ ) was conducted in a standard buffer containing $3.0 \mu \mathrm{M} \mathrm{MgCl}_{2}, 0.4 \mathrm{mM}$ dNTPs, $50 \mathrm{pmol}$ of 
each primer, $1.25 \mathrm{U}$ of MangoTaq polymerase (Bioline, London, UK) and DNA template - except for the negative (no-template) control. The cycling conditions for both primary and secondary (nested) PCRs were: $94{ }^{\circ} \mathrm{C}$ for $5 \mathrm{~min}$ (initial denaturation), followed by 35 cycles of $94{ }^{\circ} \mathrm{C}$ for $45 \mathrm{~s}$ (denaturation), $54{ }^{\circ} \mathrm{C}$ for $45 \mathrm{~s}$ (annealing) and $72{ }^{\circ} \mathrm{C}$ for $60 \mathrm{~s}$ (extension), followed by $72{ }^{\circ} \mathrm{C}$ for $10 \mathrm{~min}$ (final extension). Known test-positive, test-negative and notemplate controls were included in each PCR run.

PCR products were examined on ethidium bromidestained $1.5 \%$ agarose gels using TBE $(65 \mathrm{mM}$ Tris- $\mathrm{HCl}$, $27 \mathrm{mM}$ boric acid, $1 \mathrm{mM}$ EDTA, pH 9; Bio-Rad, Hercules, CA, USA) as the buffer and using 100 bp DNA ladder (Promega, Madison, WI, USA) as a size marker. Subsequently, secondary amplicons were individually treated with ExoSAP-IT (Affymetrix, Santa Clara, CA, USA) according to the manufacturer's instructions and directly sequenced (BigDye Terminator v.3.1 chemistry, Applied Biosystems, Foster City, CA, USA) using primers MSP-3 and MSP-4B in separate reactions. ITS sequences were aligned and analysed using the program Geneious v.10 [60] and compared with sequences from the GenBank database (Additional file 1: Table S1). Enterocytozoon bieneusi sequence types or genotypes were named according to a recent recommendation (i.e. GenBank accession number for the sequence, followed by a brief description) $[13,61]$. Sequences from the present study were submitted to the GenBank database (NCBI) and assigned specific accession numbers (MF496203 and MF496204; MF693831MF693833).

\section{Phylogenetic analysis}

Bayesian inference (BI) and Monte Carlo Markov Chain (MCMC) analysis in MrBayes v.3.2.3 [62] were used for phylogenetic analysis. Akaike Information Criteria (AIC) test in jModeltest v.2.1.7 [63] was used to evaluate the likelihood parameters set for BI analysis. Posterior probability (pp) values were calculated by running 2,000,000 generations with four simultaneous treebuilding chains, with trees being saved every 100th generation. A 50\% majority rule consensus tree for each analysis was constructed based on the final $75 \%$ of trees generated by $\mathrm{BI}$. In the phylogenetic tree, E. bieneusi clades and subclades were assigned using the classification system first proposed by Drosten et al. [51].

\section{Results}

Enterocytozoon bieneusi DNA was detected in 25 of 610 (4.1\%) faecal samples from wild deer by nested PCR-based sequencing of ITS and exclusively in sambar deer in 5 of 9 water catchment areas; the highest prevalence $(9.0 \%$; 12 of 134 samples) was recorded in catchment UY compared with $0-5.3 \%$ in the 8 other catchments (Table 1). Testpositive samples were found in all seasons, with the
Table 1 The numbers of faecal samples tested for Enterocytozoon bieneusi by nested PCR, and individual prevalences of this microsporidian in the nine of Melbourne's water catchments

\begin{tabular}{llll}
\hline Catchment & $\begin{array}{l}\text { Total no. of } \\
\text { samples tested }\end{array}$ & $\begin{array}{l}\text { No. of test-positive } \\
\text { samples }\end{array}$ & Prevalence (\%) \\
\hline Upper Yarra (UY) & 134 & 12 & 9.0 \\
Cardinia (CA) & 114 & 6 & 5.3 \\
O'Shannassay (OS) & 112 & 4 & 3.6 \\
Maroondah (MR) & 98 & 2 & 2.0 \\
Yan Yean (YY) & 113 & 1 & 0.9 \\
Armstrong (AM) & 12 & 0 & 0 \\
Silvan (SV) & 3 & 0 & 0 \\
Tarago (TAR) & 16 & 0 & 0 \\
Thompson (TH) & 8 & 0 & 0 \\
Total & 610 & 25 & 4.1 \\
\hline
\end{tabular}

highest prevalence $(6.2 \% ; 8 / 130)$ of $E$. bieneusi in spring (Table 2); prevalences varied from 1.5-16.7\% among years (Table 3).

The sequencing of all 25 ITS amplicons (243 bp) and comparisons with reference sequences in the GenBank database identified five distinct sequence types (genotypes). Of the 25 ITS sequences, 3, 1 and 1 were identical to those representing genotypes D, J and Type IV (accession numbers AF101200, AF135837 and AF242478), respectively. In addition, 19 and one ITS sequences represented two novel genotypes, designated MWC_d1 and MWC_d2, respectively, which differed at one or two of 243 nucleotide positions ( 0.41 to $0.82 \%$ difference) from the sequence with accession number KF383397 (E. bieneusi "Wildboar2"). Specifically, MWC_d1 was the dominant genotype detected in 4, 2, 2 and 11 samples from catchments CA, OS, MR and UY, respectively (Table 4).

The ITS sequence data for the five distinct genotypes (representing 25 samples) were included in a phylogenetic analysis, together with sequences representing nine established groups of E. bieneusi (Fig. 1). The major clades were well-supported ( $\mathrm{pp}=0.96$ to 01.00), but there was limited support for some clades within Group 1. Phylogenetic analysis showed that genotypes $D$ and Type IV as well as the two novel genotypes all belonged

Table 2 Seasonal prevalences of Enterocytozoon bieneusi in sambar deer in nine of Melbourne's water catchments

\begin{tabular}{llll}
\hline Season & $\begin{array}{l}\text { Total no. of } \\
\text { samples tested }\end{array}$ & $\begin{array}{l}\text { No. of test-positive } \\
\text { samples }\end{array}$ & Prevalence (\%) \\
\hline Spring & 130 & 8 & 6.2 \\
Summer & 97 & 2 & 2.1 \\
Autumn & 163 & 8 & 5.0 \\
Winter & 220 & 7 & 3.2 \\
\hline
\end{tabular}


Table 3 Annual prevalences of Enterocytozoon bieneusi in Sambar deer in nine of Melbourne's water catchment areas

\begin{tabular}{llll}
\hline Year & $\begin{array}{l}\text { Total no. of } \\
\text { samples tested }\end{array}$ & $\begin{array}{l}\text { No. of test-positive } \\
\text { samples }\end{array}$ & Prevalence (\%) \\
\hline 2009 & 57 & 1 & 1.8 \\
2010 & 60 & 3 & 5.0 \\
2011 & 67 & 1 & 1.5 \\
2012 & 54 & 2 & 3.7 \\
2013 & 67 & 2 & 3.0 \\
2014 & 118 & 10 & 8.5 \\
2015 & 67 & 2 & 3.0 \\
2016 & 114 & 3 & 2.6 \\
2017 & 6 & 1 & 16.7 \\
\hline
\end{tabular}

Table 4 Genotypes of Enterocytozoon bieneusi characterised by nested PCR-based sequencing of the internal transcribed spacer (ITS) region of the nuclear ribosomal DNA from 25 individual faecal samples from sambar deer collected (in different seasons and years) from five of Melbourne's water catchment areas

\begin{tabular}{|c|c|c|c|c|c|}
\hline Genotype designation $^{a}$ & GenBank ID & Sample code & Catchment area & Season & Year \\
\hline D & MF693831 & C1508 & Cardinia & Spring & 2010 \\
\hline D & MF693831 & UY1357 & Upper Yarra & Winter & 2010 \\
\hline D & MF693831 & YY4416 & Yan Yean & Autumn & 2014 \\
\hline J & MF693833 & OS5279 & O'Shannassay & Spring & 2014 \\
\hline Type IV & MF693832 & OS1917 & O'Shannassay & Winter & 2011 \\
\hline MWC_d1 * & MF496204 & C1505 & Cardinia & Spring & 2010 \\
\hline MWC_d1 * & MF496204 & C4255 & Cardinia & Summer & 2014 \\
\hline MWC_d1 * & MF496204 & C3645 & Cardinia & Winter & 2013 \\
\hline MWC_d1 * & MF496204 & C4857 & Cardinia & Winter & 2014 \\
\hline MWC_d1 * & MF496204 & MR7507 & Maroondah & Autumn & 2017 \\
\hline MWC_d1 * & MF496204 & MR240 & Maroondah & Winter & 2009 \\
\hline MWC_d1 * & MF496204 & OS4550 & O'Shannassay & Autumn & 2014 \\
\hline MWC_d1 * & MF496204 & OS7320 & O'Shannassay & Spring & 2016 \\
\hline MWC_d1 * & MF496204 & UY4661 & Upper Yarra & Autumn & 2014 \\
\hline MWC_d1 * & MF496204 & UY4607 & Upper Yarra & Autumn & 2014 \\
\hline MWC_d1 * & MF496204 & UY4646 & Upper Yarra & Autumn & 2014 \\
\hline MWC_d1 * & MF496204 & UY4614 & Upper Yarra & Autumn & 2014 \\
\hline MWC_d1 * & MF496204 & UY4624 & Upper Yarra & Autumn & 2014 \\
\hline MWC_d1 * & MF496204 & UY4067 & Upper Yarra & Spring & 2013 \\
\hline MWC_d1 * & MF496204 & UY6189 & Upper Yarra & Spring & 2015 \\
\hline MWC_d1 * & MF496204 & UY6181 & Upper Yarra & Spring & 2015 \\
\hline MWC_d1 * & MF496204 & UY6540 & Upper Yarra & Summer & 2016 \\
\hline MWC_d1 * & MF496204 & UY2942 & Upper Yarra & Winter & 2012 \\
\hline MWC_d1 * & MF496204 & UY6973 & Upper Yarra & Winter & 2016 \\
\hline MWC_d2 * & MF496203 & C3263 & Cardinia & Spring & 2012 \\
\hline
\end{tabular}

${ }^{a}$ Novel genotype is indicated with an asterisk to Group $1(\mathrm{pp}=0.99)$. The novel genotypes clustered with members representing Group $1 \mathrm{~b}$ without statistical support ( $\mathrm{pp}=0.90$; not shown) and genotype J clustered with representatives of Group $2(\mathrm{pp}=0.97)$.

\section{Discussion}

This is the first molecular investigation of E. bieneusi in animals in Australia. Here, we investigated the occurrence and prevalence of $E$. bieneusi in faecal samples from three species of deer ( $R$. unicolor, $C$. elaphus and $D$. dama) in nine of Melbourne's water catchment areas. Enterocytozoon bieneusi was recorded exclusively in $R$. unicolor (sambar deer) and was detected in 4.1\% (25/ 610) of this species in five of the water catchment areas, with the highest prevalence (12 of 134 samples; $9.0 \%$ ) in catchment area UY, followed by CA (5.3\%), OS (3.6\%), MR (2.0\%) and YY (0.9\%); there was no evidence of this microsporidian being present in deer in catchments AM, 


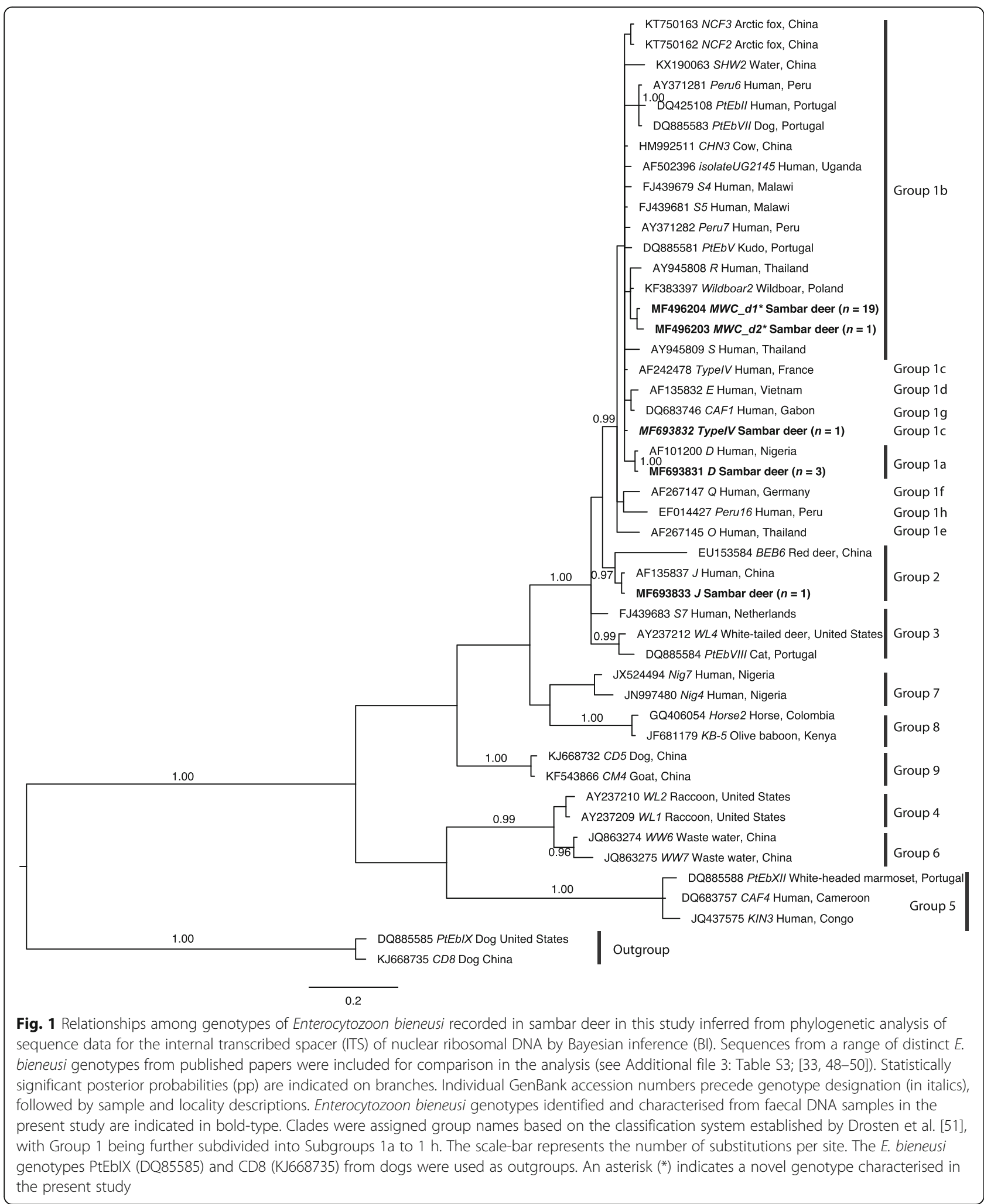

SV, TAR and TH. Enterocytozoon bieneusi was detected in sambar deer in all seasons, with the highest prevalence $(6.2 \% ; 8 / 130)$ in spring.
As environmental temperature, ultraviolet (UV) radiation and humidity are key factors that determine the survival of microsporidial spores [64-67], it is hypothesised 
that high temperature and UV radiation as well as limited rainfall limit the survival of E. bieneusi spores during summer, reducing the likelihood of transmission in this season, whereas low to moderate temperatures and UV levels as well as increased rainfall promote spore survival and transmission in spring, autumn and winter. This proposal is supported by some evidence from previous studies showing that the low prevalence of E. bieneusi in catchment areas CA and UY in summer $(2.1 \% ; 2 / 97)$ compared with other seasons was likely due to high environmental temperature [64-66] and UV exposure [67, 68] in this season. In the present study, the prevalence of E. bieneusi varied from $1.5 \%(1 / 67)$ in 2011 to $16.7 \%(1 / 6)$ in 2017 , which might relate to variability in the aforementioned environmental factors (temperature, UV and humidity) and/ or the sizes of deer populations in individual catchments, the health status of deer and aspects relating to wildlife management.

Current information about E. bieneusi of wild deer is limited to two published papers. The first is that of Guo et al. [69], who detected E. bieneusi in $12.2 \%(6 / 49)$ of wild Odocoileus virginianus (white-tailed deer) in the State of New York, USA. The second is that of Santín \& Fayer [36], who detected this microporidian in $32.5 \%(26 / 80)$ of the same deer species in Maryland, USA. Nonetheless, there are some studies of $E$. bieneusi in farmed deer from China, which involve Cervus nippon (sika deer), C. elaphus (red deer) and Elaphurus davidianus (Père David's deer) [36, 40, 69-73] (see Additional file 2: Table S2). As expected, the prevalence of E. bieneusi varied considerably (from 7.1 to $44.1 \%$ ) among different farms, which likely relates to factors such as population size of deer and density; host species, age and gender; health status and immunity of the deer; management and environmental factors - season, temperature, sunlight and humidity and stress, some of which may exert a combined effect on the survival of microsporidian species, and its transmission and prevalence. Future research might focus on long-term molecular epidemiological studies of $E$. bieneusi in different species of wild and domestic deer of differing ages and health status and in different countries to establish which environmental, climatic and/or management factors mostly influence prevalence and intensity of infection of E. bieneusi and the transmission of this microbe.

The present genetic characterisation of E. bieneusi from sambar deer revealed three known genotypes (D, J and Type IV; cf. [36, 40, 71, 73]) and two novel genotypes (MWC_d1 and MWC_d2). MWC_d1 was the predominant genotype $(76 \% ; 19 / 25)$, followed by genotypes D (12\%; 3/25), J (4\%; 1/25), MWC_d2 (4\%; 1/25) and Type IV $(4 \% ; 1 / 25)$ (i.e. J, MWC_d2 and Type IV have the same prevalence) (Table 4). Although genotypes D, J and Type IV have been detected previously in 55, 18 and 16 different species of vertebrates (see Additional file 3: Table S3) [43, 74-76], respectively, these genotypes are recorded here for the first time in sambar deer. Interestingly, the relatively broad host ranges apparent for these three genotypes (see Additional file 3: Table S3) suggest their potential to spread among different species of animals. Moreover, the presence of genotypes $\mathrm{D}, \mathrm{J}$ and Type IV in humans suggests that the animal species that harbour these genotypes represent important reservoirs for the transmission of microsporidiosis to humans. Thus, the finding of genotypes D, J and Type IV of sambar deer signals that they might be transmissible to humans. By contrast, the zoonotic potential of the two novel genotypes (MWC_d1 and MWC_d2) of E. bieneusi is presently unknown and should be explored further (indirectly) by studying E. bieneusi in humans in Melbourne, Australia, who consume unfiltered drinking water originating from Melbourne water catchments.

Sambar deer were introduced to Australia in the 1860s from the Philippines and Sri Lanka [77]. It is possible that E. bieneusi originated from these countries. However, it is also possible that sambar deer acquired infection following introduction into Australia. The origin cannot yet be inferred due to the absence of data for $E$. bieneusi in sambar deer and other animals in those two countries. Further studies would be needed to attempt to establish the origin of E. bieneusi. The transmission of E. bieneusi has been proposed to occur mainly through contaminated food, and drinking, recreational and waste waters $[25-27,78]$. All three known genotypes (D, J and Type IV) identified here in sambar deer have also been detected previously in water in China, Ireland, Nigeria, Spain and Tunisia [25, 26, 79-85] (see Additional file 3: Table S3), indicating that E. bieneusi-contaminated water might be a key source for transmission of microsporidiosis to various species of animals including humans. This information is a stimulus to explore whether $E$. bieneusi is present in Melbourne's water.

Phylogenetic analysis of ITS gene sequence data sets have shown previously that genotype $J$ belongs to a 'cattle-specific' assemblage (called Group 2; [86]), whereas genotypes D, MWC_d1, MWC_d2 and Type IV identified in the present study are inferred to be in the 'zoonotic' assemblage (called Group 1) (Fig. 1). Therefore, it would be interesting to extend studies of E. bieneusi to humans in Melbourne and environs, and other species of animals in Melbourne's water catchments, but perhaps, more importantly, to investigate the presence of E. bieneusi in catchment-source and drinking water in Melbourne, Australia. Also, further studies could explore other genetic markers for genotypic characterization of E. bieneusi to establish whether a genotype defined by the ITS region is in accord with assignment using a small set of other genetic markers, 
or a large panel of, for example, single copy genes from the E. bieneusi genome.

Since E. bieneusi can cause acute or chronic enteritis and sometimes systemic disease [18-21], this microsporidian species has been classified as a Category B Priority Pathogen by the National Institute of Allergy and Infectious Diseases (NIAID) [33, 87], although there is no reference to E. bieneusi in the National Water Quality Management Strategy (NWQMS) of Australia or the Australian Drinking Water Guidelines (ADWG) contained within this strategy [53]. Given the lack of studies of E. bieneusi in Australia, it would seem prudent to gain insights into the epidemiology of this parasite in wildlife, water and the environment in Australia, to work toward an informed position on the public health significance of microsporidiosis associated with $E$. bieneusi. It would also be relevant to explore whether E. bieneusi survives currently used water treatment protocols [53].

\section{Conclusions}

This study records for the first time $E$. bieneusi in sambar deer in Australia. The genotypes of E. bieneusi (D, J and Type IV) identified here have been detected previously in humans and water samples in other countries, which suggests that this deer species might act as a reservoir for genotypes that are transmissible to humans. As nothing is known about the occurrence, prevalence or distribution of E. bieneusi in other animals in Australia or its significance as a pathogen, future studies should elucidate the epidemiology of E. bieneusi in wildlife, water and the environment, in order to provide an informed position on its public health importance in this country. In the first instance, it would be important to focus on investigating whether other animals that are abundant in Melbourne's water catchments carry $E$. bieneusi genotypes reported in the literature to infect humans. Other studies could be conducted to establish whether some of the genotypes recognised to be potentially zoonotic actually occur in humans in Australia.

\section{Additional files}

Additional file 1: Table S1. Enterocytozoon bieneusi genotypes recorded from different animal species and water samples from published literature, representing nine distinct groups and two outgroups. The genotypes of E. bieneusi identified in this study are listed at the end of this table. (DOCX $74 \mathrm{~kb}$ )

Additional file 2: Table S2. Genotypes of Enterocytozoon bieneusi recorded previously in five species of deer worldwide and identified in sambar deer in the present study. (DOCX $69 \mathrm{~kb}$ )

Additional file 3: Table S3. Genotypes D, J and Type IV of Enterocytozoon bieneusi recorded in different animal species and water samples in previous studies. These genotypes were also recorded in the present study. (DOCX $359 \mathrm{~kb})$

\section{Abbreviations}

AIC: Akaike information criteria; BI: Bayesian inference; ITS: Internal transcribed spacer of nuclear ribosomal DNA; LSU: Large subunit of nuclear ribosomal RNA gene; MCMC: Monte Carlo Markov Chain; pp: Posterior probability; SSU: Small subunit of nuclear ribosomal RNA gene

\section{Acknowledgements}

Melbourne Water staff are gratefully acknowledged.

\section{Funding}

Research funding from the Melbourne Water Corporation and Australian Research Council (grant number LP160101299) (RBG and AVK) is gratefully acknowledged. Yan Zhang was the recipient of scholarships from the Chinese Scholarship Council (CSC) and The University of Melbourne.

\section{Availability of data and materials}

Nucleotide sequences reported in this paper are available in the GenBank database under accession numbers MF496203 and MF496204 and MF693831-MF693833.

\section{Authors' contributions}

Collected samples: AVK and SRH. Analysis and interpretation: YZ, AVK, TW and RBG. Wrote the paper: YZ, AVK and RBG. Supervision of project: AVK, TW and RBG. Grant funding: RBG and AVK. All authors read and approved the final manuscript.

Ethics approval and consent to participate

Not applicable.

Consent for publication

Not applicable.

\section{Competing interests}

The authors declare that they have no competing interests.

\section{Publisher's Note}

Springer Nature remains neutral with regard to jurisdictional claims in published maps and institutional affiliations.

\section{Author details}

'Department of Veterinary Biosciences, Melbourne Veterinary School, Faculty of Veterinary and Agricultural Sciences, The University of Melbourne, Parkville, VIC 3010, Australia. ${ }^{2}$ Melbourne Water, Docklands, VIC 3001, Australia.

Received: 30 August 2017 Accepted: 6 December 2017

Published online: 03 January 2018

\section{References}

1. Mathis A, Weber R, Deplazes P. Zoonotic potential of the microsporidia. Clin Microbiol Rev. 2005;18(3):423-45.

2. Anane S, Attouchi H. Microsporidiosis: epidemiology, clinical data and therapy. Gastroenterol Clin Biol. 2010;34(8):450-64.

3. Capella-Gutiérrez S, Marcet-Houben M, Gabaldón T. Phylogenomics supports microsporidia as the earliest diverging clade of sequenced fungi. BMC Biol. 2012;10(1):47.

4. Keeling P. Five questions about Microsporidia. PLoS Pathog. 2009;5(9):e1000489.

5. Adl SM, Simpson AG, Farmer MA, Andersen RA, Anderson OR, Barta JR, et al. The new higher level classification of eukaryotes with emphasis on the taxonomy of protists. J Eukaryot Microbiol. 2005;52(5):399-451.

6. James TY, Kauff F, Schoch CL, Matheny PB, Hofstetter V, Cox CJ, et al. Reconstructing the early evolution of fungi using a six-gene phylogeny. Nature. 2006;443(7113):818-22.

7. Katinka MD, Duprat $\mathrm{S}$, Cornillot E, Méténier G, Thomarat F, Prensier G, et al. Genome sequence and gene compaction of the eukaryote parasite Encephalitozoon cuniculi. Nature. 2001;414(6862):450-3.

8. Vávra J, Lukeš J. Microsporidia and 'the art of living together'. Adv Parasitol. 2013;82:253-319.

9. Weiss LM, Vossbrinck CR. Molecular biology, molecular phylogeny, and molecular diagnostic approaches to the microsporidia. In: Murray W, Weiss 
LM, editors. The microsporidia and microsporidiosis. Washington, D.C., USA: American Society of Microbiology; 1999, pp. 129-71.

10. Fayer R, Santín-Durán M. Epidemiology of microsporidia in human infections. In: Weiss L, Becnel JJ, editors. Microsporidia: pathogens of opportunity. Chichester, UK: John Wiley \& Sons; 2014. p. 135-46.

11. Desportes I, Le Charpentier Y, Galian A, Bernard F, Cochand-Priollet B, Lavergne A, et al. Occurrence of a new microsporidan: Enterocytozoon bieneusi n. G., n. Sp., in the enterocytes of a human patient with AIDS. J Protozool. 1985;32(2):250-4

12. Santín-Durán M. Enterocytozoon bieneusi. In: Xiao L, Ryan U, Feng Y, editors. Biology of foodborne parasites. Boca Raton, FL, USA: CRC Press; 2015. p. 149-74.

13. Santín M, Fayer R. Microsporidiosis: Enterocytozoon bieneusi in domesticated and wild animals. Res Vet Sci. 2011;90(3):363-71.

14. Stark D, van Hal S, Barratt J, Ellis J, Marriott D, Harkness J. Limited genetic diversity among genotypes of Enterocytozoon bieneusi strains isolated from HIV-infected patients from Sydney, Australia. J Med Microbiol. 2009;58(3):355-7.

15. Espern A, Morio F, Miegeville M, Illa H, Abdoulaye M, Meyssonnier V, et al. Molecular study of microsporidiosis due to Enterocytozoon bieneusi and Encephalitozoon intestinalis among human immunodeficiency virus-infected patients from two geographical areas: Niamey, Niger, and Hanoi, Vietnam. J Clin Microbiol. 2007;45(9):2999-3002.

16. Akinbo FO, Okaka CE, Omoregie R, Dearen T, Leon ET, Xiao L. Molecular epidemiologic characterization of Enterocytozoon bieneusi in HIV-infected persons in Benin City, Nigeria. Am J Trop Med Hyg. 2012;86(3):441-5.

17. Lobo ML, Xiao L, Antunes F, Matos O. Microsporidia as emerging pathogens and the implication for public health: a 10-year study on HIV-positive and -negative patients. Int J Parasitol. 2012;42(2):197-205.

18. Molina JM, Sarfati C, Beauvais B, Lémann M, Lesourd A, Ferchal F, et al. Intestinal microsporidiosis in human immunodeficiency virus-infected patients with chronic unexplained diarrhea: prevalence and clinical and biologic features. J Infect Dis. 1993;167(1):217-21.

19. Weber R, Bryan RT, Schwartz DA, Owen RL. Human microsporidial infections. Clin Microbiol Rev. 1994;7(4):426-61.

20. Didier ES, Weiss LM. Microsporidiosis: current status. Curr Opin Infect Dis. 2006;19(5):485-92.

21. Kotler DP, Orenstein JM. Clinical syndromes associated with microsporidiosis. Adv Parasitol. 1998;40:321-49.

22. Cotte L, Rabodonirina M, Chapuis F, Bailly F, Bissuel F, Raynal C, et al. Waterborne outbreak of intestinal microsporidiosis in persons with and without human immunodeficiency virus infection. J Infect Dis. 1999; 180(6):2003-8

23. Nkinin SW, Asonganyi T, Didier ES, Kaneshiro ES. Microsporidian infection is prevalent in healthy people in Cameroon. J Clin Microbiol. 2007;45(9):2841-6.

24. Sak $B$, Brady D, Pelikánová $M$, Květoňová $D$, Rost $M$, Kostka $M$, et al. Unapparent microsporidial infection among immunocompetent humans in the Czech Republic. J Clin Microbiol. 2011;49(3):1064-70.

25. Ayed LB, Yang W, Widmer G, Cama V, Ortega Y, Xiao L. Survey and genetic characterization of wastewater in Tunisia for Cryptosporidium spp., Giardia duodenalis, Enterocytozoon bieneusi, Cyclospora cayetanensis and Eimeria spp. J Water Health. 2012;10(3):431-44.

26. Galván AL, Magnet A, Izquierdo F, Fenoy S, Rueda C, Fernandez Vadillo C, et al. Molecular characterization of human-pathogenic microsporidia and Cyclospora cayetanensis isolated from various water sources in Spain: a year-long longitudinal study. Appl Environ Microbiol. 2013;79(2): 449-59.

27. Decraene V, Lebbad M, Botero-Kleiven S, Gustavsson AM, Löfdahl M. First reported foodborne outbreak associated with microsporidia, Sweden, October 2009. Epidemiol Infect. 2012;140(3):519-27.

28. Jedrzejewski S, Graczyk TK, Slodkowicz-Kowalska A, Tamang L, Majewska AC. Quantitative assessment of contamination of fresh food produce of various retail types by human-virulent microsporidian spores. Appl Environ Microbiol. 2007;73(12):4071-3

29. Botterel F, Minozzi C, Vittecoq D, Bourée P. Pulmonary localization of Enterocytozoon bieneusi in an AIDS patient: case report and review. J Clin Microbiol. 2002;40(12):4800-1.

30. del Aguila C, Lopez-Velez R, Fenoy S, Turrientes C, Cobo J, Navajas R, et al. Identification of Enterocytozoon bieneusi spores in respiratory samples from an AIDS patient with a 2-year history of intestinal microsporidiosis. J Clin Microbiol. 1997;35(7):1862-6.
31. Weber R, Kuster H, Keller R, Bächi T, Spycher MA, Briner J, et al. Pulmonary and intestinal microsporidiosis in a patient with the acquired immunodeficiency syndrome. Am Rev Respir Dis. 1992;146:1603-5.

32. Graczyk TK, Sunderland D, Rule AM, da Silva AJ, Moura IN, Tamang L, et al. Urban feral pigeons (Columba livia) as a source for air- and waterborne contamination with Enterocytozoon bieneusi spores. Appl Environ Microbiol. 2007;73(13):4357-8.

33. Karim MR, Wang R, Dong H, Zhang L, Li J, Zhang S, et al. Genetic polymorphism and zoonotic potential of Enterocytozoon bieneusi fom non-human primates in China. Appl Environ Microbiol. 2014;80(6):1893-8.

34. Santín M, Cortes Vecino JA, Fayer R. Enterocytozoon bieneusi genotypes in dogs in Bogota, Colombia. Am J Trop Med Hyg. 2008;79(2):215-7.

35. Santín M, Dargatz D, Fayer R. Prevalence and genotypes of Enterocytozoon bieneusi in weaned beef calves on cow-calf operations in the USA. Parasitol Res. 2012;110(5):2033-41.

36. Santín M, Fayer R. Enterocytozoon bieneusi, Giardia, and Cryptosporidium infecting white-tailed deer. J Eukaryot Microbiol. 2015;62(1):34-43.

37. Ma J, Li P, Zhao X, Xu H, Wu W, Wang Y, et al. Occurrence and molecular characterization of Cryptosporidium spp. and Enterocytozoon bieneusi in dairy cattle, beef cattle and water buffaloes in China. Vet Parasitol. 2015;207(3):220-7.

38. Fiuza VR, Oliveira FC, Fayer R, Santín M. First report of Enterocytozoon bieneusi in pigs in Brazil. Parasitol Int. 2015;64(4):18-23.

39. Wan Q, Lin Y, Mao Y, Yang Y, Li Q, Zhang S, et al. High prevalence and widespread distribution of zoonotic Enterocytozoon bieneusi genotypes in swine in northeast China: implications for public health. J Eukaryot Microbiol. 2016;63(2):162-70.

40. Zhang XX, Cong W, Liu GH, Ni XT, Ma JG, Zheng WB, et al. Prevalence and genotypes of Enterocytozoon bieneusi in sika deer in Jilin province, northeastern China. Acta Parasitol. 2016;61(2):382-8.

41. Zhang XX, Jiang J, Cai YN, Wang CF, Xu P, Yang GL, et al. Molecular characterization of Enterocytozoon bieneusi in domestic rabbits (Oryctolagus cuniculus) in northeastern China. Korean J Parasitol. 2016;54(1):81-5.

42. Wan Q, Xiao L, Zhang X, Li Y, Lu Y, Song M, et al. Clonal evolution of Enterocytozoon bieneusi populations in swine and genetic differentiation in subpopulations between isolates from swine and humans. PLoS Negl Trop Dis. 2016;10(8):e0004966.

43. da Cunha MJR, Cury MC, Santín M. Molecular identification of Enterocytozoon bieneusi, Cryptosporidium, and Giardia in Brazilian captive birds. Parasitol Res. 2017;116(2):487-93.

44. Khanduja S, Ghoshal U, Ghoshal UC. Phylogenetic analysis of genetically distinct Enterocytozoon bieneusi infecting renal transplant recipients. Acta Parasitol. 2017;62(1):63-8.

45. Yu F, Wu Y, Li T, Cao J, Wang J, Hu S, et al. High prevalence of Enterocytozoon bieneusi zoonotic genotype $D$ in captive golden snub-nosed monkey (Rhinopithecus roxellanae) in zoos in China. BMC Vet Res. 2017;13(1):158.

46. Baroudi D, Khelef D, Hakem A, Abdelaziz A, Chen X, Lysen C, et al. Molecular characterization of zoonotic pathogens Cryptosporidium spp., Giardia duodenalis and Enterocytozoon bieneusi in calves in Algeria. Vet Parasitol. 2017:8:66-9.

47. Qi M, Jing B, Jian FC, Wang RJ, Zhang SM, Wang HY, et al. Dominance of Enterocytozoon bieneusi genotype $J$ in dairy calves in Xinjiang, northwest China. Parasitol Int. 2017;66(1):960-3.

48. Li W, Li Y, Song M, Lu Y, Yang J, Tao W, et al. Prevalence and genetic characteristics of Cryptosporidium, Enterocytozoon bieneusi and Giardia duodenalis in cats and dogs in Heilongjiang province, China. Vet Parasitol. 2015;208(3):125-34.

49. Karim MR, Dong H, Li T, Yu F, Li D, Zhang L, et al. Predomination and new genotypes of Enterocytozoon bieneusi in captive nonhuman primates in zoos in China: high genetic diversity and zoonotic significance. PLoS One. 2015;10(2):e0117991.

50. Thellier M, Breton J. Enterocytozoon bieneusi in human and animals, focus on laboratory identification and molecular epidemiology. Parasite. 2008;15(3):349-58.

51. Drosten C, Laabs J, Kuhn EM, Schottelius J. Interspecies transmission of Enterozytozoon bieneusi supported by observations in laboratory animals and phylogeny. Med Microbiol Immunol. 2005;194(4):207-9.

52. Field AS, Hing MC, Milliken ST, Marriott DJ. Microsporidia in the small intestine of HIV-infected patients. A new diagnostic technique and a new species. Med J Aust. 1993;158(6):390-4. 
53. ADWG. Australian drinking water guidelines 6. Version 3.3. 2011. https:// www.nhmrc.gov.au/guidelines/publications/eh52. Accessed August 2017.

54. WHO. Guidelines for Drinking-Water Quality. Fourth edition. Geneva: World Health Organization; 2011.

55. Koehler AV, Bradbury RS, Stevens MA, Haydon SR, Jex AR, Gasser RB. Genetic characterization of selected parasites from people with histories of gastrointestinal disorders using a mutation scanning-coupled approach. Electrophoresis. 2013;34(12):1720-8.

56. Nolan MJ, Jex AR, Koehler AV, Haydon SR, Stevens MA, Gasser RB. Molecularbased investigation of Cryptosporidium and Giardia from animals in water catchments in southeastern Australia. Water Res. 2013;47(5):1726-40.

57. Triggs B. Tracks, scats and other traces: a field guide to Australian mammals. Melbourne: Oxford University Press; 1996.

58. Dalén L, Götherström A, Angerbjörn A. Identifying species from pieces of faeces. Conserv Genet. 2004;5(1):109-11.

59. Katzwinkel-Wladarsch S, Lieb M, Helse W, Löscher T, Rinder H. Direct amplification and species determination of microsporidian DNA from stool specimens. Tropical Med Int Health. 1996;1(3):373-8.

60. Kearse M, Moir R, Wilson A, Stones-Havas S, Cheung M, Sturrock S, et al. Geneious basic: an integrated and extendable desktop software platform for the organization and analysis of sequence data. Bioinformatics. 2012;28(12):1647-9.

61. Santín M, Fayer R. Enterocytozoon bieneusi genotype nomenclature based on the internal transcribed spacer sequence: a consensus. J Eukaryot Microbiol. 2009;56(1):34-8.

62. Huelsenbeck JP, Ronquist FMRBAYES. Bayesian inference of phylogenetic trees. Bioinformatics. 2001;17(8):754-5.

63. Darriba D, Taboada GL, Doallo R, Posada D. jModelTest 2: more models, new heuristics and parallel computing. Nat Methods. 2012;9(8):772.

64. Li X, Palmer R, Trout JM, Fayer R. Infectivity of microsporidia spores stored in water at environmental temperatures. J Parasitol. 2003;89(1):185-8.

65. Kramer JP. Longevity of microsporidian spores with special reference to Octosporea muscaedomesticae flu. Acta Protozool. 1970;8(17):217-24.

66. Koudela B, Kučerová Š, Hudcovic T. Effect of low and high temperatures on infectivity of Encephalitozoon cuniculi spores suspended in water. Folia Parasitol. 1999;46(3):171-4.

67. Kelly JF, Anthony DW. Susceptibility of spores of the microsporidian Nosema algerae to sunlight and germicidal ultraviolet radiation. J Invertebr Pathol. 1979:34(2):164-9.

68. Kaya HK. Survival of spores of Vairimorpha (= Nosema) necatrix (Microsporida: Nosematidae) exposed to sunlight, ultraviolet radiation, and high temperature. J Invertebr Pathol. 1977;30(2):192-8.

69. Guo Y, Alderisio KA, Yang W, Cama V, Feng Y, Xiao L. Host specificity and source of Enterocytozoon bieneusi genotypes in a drinking source watershed. Appl Environ Microbiol. 2014;80(1):218-25.

70. Li W, Deng L, Yu X, Zhong Z, Wang Q, Liu X, et al. Multilocus genotypes and broad host-range of Enterocytozoon bieneusi in captive wildlife at zoological gardens in China. Parasit Vectors. 2016;9(1):395.

71. Zhang Z, Huang J, Karim MR, Zhao J, Dong H, Ai W, et al. Zoonotic Enterocytozoon bieneusi genotypes in Père David's deer (Elaphurus davidianus) in Henan, China. Exp Parasitol. 2015;155:46-8.

72. Zhao W, Zhang W, Wang R, Liu W, Liu A, Yang D, et al. Enterocytozoon bieneusi in sika deer (Cervus nippon) and red deer (Cervus elaphus): deer specificity and zoonotic potential of ITS genotypes. Parasitol Res. 2014;113(11):4243-50.

73. Huang J, Zhang Z, Yang Y, Wang R, Zhao J, Jian F, et al. New genotypes of Enterocytozoon bieneusi isolated from sika deer and red deer in China. Front Microbiol. 2017:8:879.

74. Sadler F, Peake N, Borrow R, Rowl PL, Wilkins EG, Curry A. Genotyping of Enterocytozoon bieneusi in AIDS patients from the north west of England J Inf Secur. 2002;44(1):39-42.

75. Lee $\mathrm{JH}$. Prevalence and molecular characteristics of Enterocytozoon bieneusi in cattle in Korea. Parasitol Res. 2007;101(2):391-6.

76. Reetz J, Rinder H, Thomschke A, Manke H, Schwebs M, Bruderek A. First detection of the microsporidium Enterocytozoon bieneusi in non-mammalian hosts (chickens). Int J Parasitol. 2002;32(7):785-7.

77. Bentley A. An introduction to the deer of Australia with special reference to Victoria., 3rd edn. Melbourne: Australian Deer Research Foundation; 1998.

78. Graczyk TK, Lucy FE, Tamang L, Miraflor A. Human enteropathogen load in activated sewage sludge and corresponding sewage sludge end products. Appl Environ Microbiol. 2007;73(6):2013-5.

79. Li N, Xiao L, Wang L, Zhao S, Zhao X, Duan L, et al. Molecular surveillance of Cryptosporidium spp., Giardia duodenalis, and Enterocytozoon bieneusi by genotyping and subtyping parasites in wastewater. PLoS Negl Trop Dis. 2012:6(9):e1809.

80. Graczyk TK, Lucy FE, Mashinsky Y, Andrew Thompson RC, Koru O, daSilva AJ. Human zoonotic enteropathogens in a constructed free-surface flow wetland. Parasitol Res. 2009;105(2):423-8.

81. Ye J, Xiao L, Ma J, Guo M, Liu L, Feng Y. Anthroponotic enteric parasites in monkeys in public park, China. Emerg Infect Dis. 2012;18(10):1640-3.

82. Ma J, Feng Y, Hu Y, Villegas EN, Xiao L. Human infective potential of Cryptosporidium spp., Giardia duodenalis and Enterocytozoon bieneusi in urban wastewater treatment plant effluents. J Water Health. 2016;14(3):411-23.

83. Ojuromi OT, Duan L, Izquierdo F, Fenoy SM, Oyibo WA, Del Aguila C, et al, Genotypes of Cryptosporidium spp. and Enterocytozoon bieneusi in human immunodeficiency virus-infected patients in Lagos, Nigeria. J Eukaryot Microbiol. 2016;63(4):414-8.

84. Ye J, Yan J, Xu J, Ma K, Yang X. Zoonotic Enterocytozoon bieneusi in raw wastewater in Zhengzhou, China. Folia Parasitol (Praha). 2017;64:002.

85. Huang C, Hu Y, Wang L, Wang Y, Li N, Guo Y, et al. Environmental transport of emerging human-pathogenic Cryptosporidium species and subtypes through combined sewer overflow and wastewater. Appl Environ Microbiol. 2017; https://doi.org/10.1128/AEM.00682-17.

86. Sulaiman IM, Fayer R, Yang C, Santín M, Matos O, Xiao L. Molecular characterization of Enterocytozoon bieneusi in cattle indicates that only some isolates have zoonotic potential. Parasitol Res. 2004;92(4):328-34.

87. Akiyoshi DE, Morrison HG, Lei S, Feng X, Zhang Q, Corradi N, et al. Genomic survey of the non-cultivatable opportunistic human pathogen, Enterocytozoon bieneusi. PLoS Pathog. 2009;5(1):e1000261.

\section{Submit your next manuscript to BioMed Central and we will help you at every step:}

- We accept pre-submission inquiries

- Our selector tool helps you to find the most relevant journal

- We provide round the clock customer support

- Convenient online submission

- Thorough peer review

- Inclusion in PubMed and all major indexing services

- Maximum visibility for your research

Submit your manuscript at www.biomedcentral.com/submit
) Biomed Central 